\title{
Output Injection Filtering Redesign in High-Gain Observers
}

\author{
Daniele Astolfi ${ }^{1}$, Marc Jungers ${ }^{1}$ and Luca Zaccarian ${ }^{2}$
}

\begin{abstract}
We propose a new paradigm to redesign high-gain observers in order to improve performances in the presence of measurement noise. In particular, instead of driving the observer by means of a standard output injection term, we filter it with a dynamical system having good filtering properties. In this first preliminary result we also select the filter in order to address numerical challenges.

Index Terms - High-gain observers, noise filtering.
\end{abstract}

\section{INTRODUCTION}

Among different techniques for the state-estimation of continuous-time nonlinear systems, high-gain observers are certainly very popular in nonlinear control theory. After seminal works at the end of the 80's, a large number of researchers devoted their attention to the study of this class of observers, see, for instance, [1]-[3] and references therein. The main advantage of a high-gain observer is that it guarantees that the estimation error converges exponentially to zero with a rate of convergence that can be arbitrarily augmented by enlarging just one single parameter, denoted as highgain parameter. However, because of the fast convergence, sensitivity to high-frequency measurement noise is made worst, see [4]-[6]. To address this problem, many techniques have been developed in the last decades: adaptive techniques, [7], [8], redesign of the local behaviour by combining different observers, [9]-[11], low-power structures, [12]-[16], and lastly saturation redesign, [17]. In the recent work [18], it is proposed to filter the measured output by means of a fast low-pass filter, that is a low-pass filter which is modulated by the high-gain parameter. The main drawback of this approach, however, is that asymptotic (possibly practical in presence of measurement noise) estimation cannot be any more guaranteed and the proposed scheme guarantees good performances only with feedback stabilization. Therefore, in the case where we are interested in purely estimation, that scheme can not be used any longer.

In this work, inspired by [12] and in the same spirit [18], we propose to use a low-pass filter in order to improve performances of high-gain observers in the presence of measurement noise while retaining the main behaviours. In the proposed design, the dynamical filter processes the output injection term of the observer and not the measured output, as in [18]. Sufficient conditions for design of the matrix coefficients, ensuring convergence of the observer, are

\footnotetext{
1 Université de Lorraine, CNRS, CRAN, F-54000 Nancy, France \{daniele.astolfi@univ-lorraine.fr, marc.jungers@univ-lorraine.fr\}.

2 LAAS-CNRS, Université de Toulouse, CNRS, Toulouse, France, and Dep. of Industrial Engineering, University of Trento, Trento, Italy zzaccarianelaas.fr\}.

This project has received funding from the "Région Grand-Est" and from CaRiTRo, grant PowerLyap.
}

provided. The estimation error provided by the new structure retains the same input-to-state properties of standard highgain observers. A special choice of this dynamical filter is also given, where we exploit the possibility of writing all the coefficients embedding powers of the high-gain parameter of order 1 and 2 instead of $n$, similarly to the low-power structures presented in [12]-[16]. In this case, the choice of the matrix coefficients must satisfy a necessary condition, which is fully exploited. With respect to [12]-[15], the procedure of selection of the coefficients in the proposed structure is much simpler. Simulations are enclosed at the end of the work.

We highlight that the goal of this preliminary work is to present a new structure for the design of high-gain observer that aim to address the problem of state observation in noisy frameworks. For this reason, we are not going to compare the proposed techniques with other existing high-gain observer techniques (such as [12], [18]) but we will show that dynamical filtering may be effective in improving disturbance rejection properties while inheriting the asymptotic behaviours of the observers. Optimalitybased tuning for both the gain and the parameters of the dynamical filter remains an open question for future research.

Notation. We denote with $\mathbb{R}$ the set of real numbers and with $\mathbb{Z}$ the set of integers. We denote also $\mathbb{Z}_{>0}=\{1,2, \ldots\}$. We denote with $\mathbb{C}$ the set of complex numbers and $\mathbb{C}_{<0}$ the set of complex numbers with strictly negative real part. We denote with $i$ the imaginary number. Given a complex number $z \in \mathbb{C}, z=x+i y$, with $x, y \in \mathbb{R}$, we denote with $\Re(z)$ its real part, namely $\Re(z)=x$ and $\Im(z)$ its imaginary part, that is $\Im(z)=y$. Given a square matrix $F$, we define with $\sigma(F)$ the spectrum of $F$ and with $p_{F}(\lambda)$ its characteristic polynomial, namely $p_{F}(\lambda)=\operatorname{det}(\lambda I-F)$. We denote with $I_{i}$, or simply $I$, an identity matrix of dimension $i \in \mathbb{Z}_{>0}$. We denote with $0_{i, j}$ a zero matrix of dimension $i \times j$, with $i, j \in \mathbb{Z}_{>0}$. We denote a triplet in prime form $\left(A_{i}, B_{i}, C_{i}\right)$ of dimension $i \in \mathbb{Z}_{>0}$, (or simply $(A, B, C)$ in the case of no dimension ambiguity), matrices of the form

$$
A_{i}=\left(\begin{array}{cc}
0_{i-1,1} & I_{i-1} \\
0 & 0_{1, i-1}
\end{array}\right), \quad B_{i}=\left(\begin{array}{c}
0_{i-1,1} \\
1
\end{array}\right), C_{i}^{T}=\left(\begin{array}{c}
1 \\
0_{i-1,1}
\end{array}\right) \text {. }
$$

\section{Preliminaries}

In this work we consider nonlinear autonomous systems of the form

$$
\dot{x}(t)=A x(t)+B \varphi(x(t)), \quad y(t)=C x(t)+\nu(t),
$$

where $x \in \mathbb{R}^{n}$ is the state, $n \in \mathbb{Z}_{>0}, y \in \mathbb{R}$ is the measured output, $\nu: \mathbb{R}_{\geq 0} \rightarrow \mathbb{R}$ is some (bounded) measurement noise, 
$(A, B, C)$ is a triplet in prime form, see (1), of dimension $n$ and the function $\varphi(\cdot)$ is at least locally Lipschitz. Note that the class (2) represents a fairly general class of systems (e.g. all linear observable and controllable dynamics) due to the general function $\varphi(\cdot)$. Moreover, we recall that any observable time-invariant autonomous nonlinear system can be written in the form (2) after a suitable change of coordinates, see [1]-[3] and references therein. We suppose that the state $x$ of (2) evolves in a given compact set $X \subset \mathbb{R}^{n}$ for all $t \geq 0$. In the rest of the paper we will drop the time-dependency $(t)$ on the variable when not useful. High-gain observers for system (2) can be designed as

$$
\dot{\hat{x}}=A \hat{x}+B \varphi_{s}(\hat{x})+D_{k} L(y-C \hat{x})
$$

where $\hat{x} \in \mathbb{R}^{n}$ is the state of the observer, $D_{k}=$ $\operatorname{diag}\left(k, \ldots, k^{n}\right)$, where $k \geq 1$ is a real number usually denoted as high-gain parameter, $L=\operatorname{col}\left(\ell_{1}, \ldots, \ell_{n}\right)$ is the Luenberger gain to be chosen such that $(A-L C)$ is Hurwitz, and $\varphi_{s}$ is a globally bounded and locally Lipschitz function that agrees with $\varphi$ on $X$, namely $\varphi_{s}(x)=\varphi(x)$ for all $x \in X$ and there exists a number $\bar{\varphi}>0$ such that $\left|\varphi_{s}(x)\right| \leq \bar{\varphi}$ for all $x \in \mathbb{R}^{n}$. For instance, $\varphi$ can be designed by saturating the function $\varphi$ outside the compact set $X$. By using the fact that the matrix $(A-L C)$ is Hurwitz, it can be shown that, for a $k$ sufficiently large, the estimation error $\hat{x}-x$ satisfies the following bound

$$
|\hat{x}(t)-x(t)| \leq k^{n-1} \exp \left(-a_{1} k t\right) a_{2}+a_{3} k^{n-1} \sup _{t \geq 0}|\nu(t)|
$$

where $a_{i}>0, i=1,2,3$ are numbers independent of $k$. Bound (4) highlights that the estimation error converges asymptotically to zero when the measurement noise is not present. Moreover, observer (3) has good asymptotic properties with respect to the measurement noise (they can also be denoted as ISS-gain). On the other hand, the gain between the measurement noise and the estimation error is in general proportional to $k^{n-1}$ showing that the faster is the observer, the worst will be in general the performances in the presence of measurement noise. As shown in [6], the low-pass filter behaviour of the high-gain observer (3) can be captured in case the measurement noise $\nu(t)$ is modelled by a finite Fourier series, namely

$$
\nu(t):=\sum_{i=1}^{N} \nu_{i} \sin \left(\frac{\omega_{i}}{\epsilon} t+\phi_{i}\right),
$$

for some $N>0$, where the parameter $\epsilon>0$ parametrizes the basic frequencies $\omega_{i}$ while $\nu_{i}>0$ and $\phi_{i}$ characterize the amplitude and the phase of the single components, respectively. The measurement noise (5) has a high-frequency behaviour for $\epsilon$ small enough. In this case, the asymptotic estimation error in (4) can be computed as

$$
\lim _{t \rightarrow \infty}|\hat{x}(t)-x(t)| \leq \epsilon a_{4} k^{n} \sup _{t \geq 0}|\nu(t)| \leq \epsilon a_{4} k^{n} \sup _{t \geq 0} \sum_{i} \nu_{i}
$$

where $a_{4}>0$ is a number independent of $k$. It is readily seen that the bound (6) catches the low-pass filter behaviour of the high-gain observer (3) since

$$
\lim _{\epsilon \rightarrow 0} \lim _{t \rightarrow \infty}|\hat{x}(t)-x(t)|=0 .
$$

On the other hand, bound (6) highlights that the asymptotic gain at high-frequencies is proportional to $k^{n}$ and not to $k^{n-1}$. Nevertheless, as showed in [15], it can be shown that augmenting the relative degree between the measured output and the state estimations, may improve the asymptotic estimation (6). For this reason, in this work, we propose to redesign the observer (3) by augmenting the relative degree between the measured output and the estimates. This task is accomplished by filtering the output injection term through a dynamical linear filter.

\section{MAin Results}

\section{A. Observer structure}

The high-gain observer (3) can be represented by a blockdiagram scheme as in Figure 1. The main objective of this work is to modify observer (3) by adding a suitable filter between the output injection term and the observer, as in Figure 2. In particular, in this section, we study a high-gain observer with filtering redesign of the form

$$
\begin{aligned}
\dot{\hat{x}}_{i} & =\hat{x}_{i+1}+\ell_{i} \eta_{i}, & i=1, \ldots, n-1 \\
\dot{\hat{x}}_{n} & =\varphi_{s}(\hat{x})+\ell_{n} \eta_{n}, & \\
\dot{\eta}_{1} & =-k \alpha \eta_{1}+k^{2} \beta\left(y-\hat{x}_{1}\right), & \\
\dot{\eta}_{j} & =-k \alpha \eta_{j}+k^{2} \beta \eta_{j-1}, & j=2, \ldots, n,
\end{aligned}
$$

where $(\hat{x}, \eta) \in \mathbb{R}^{2 n}$ is the extended state, $\varphi_{s}$ is chosen as for the standard high-gain observer (3), and $\alpha, \beta, \ell_{1}, \ldots, \ell_{n}$ are positive coefficients to be chosen. We denote with $\widehat{X} \times E \subset$ $\mathbb{R}^{n} \times \mathbb{R}^{n}$ the compact set of initial conditions of (7). By inspecting the $\eta$-dynamics in (7), it can be noticed that $\eta_{i}$ plays the role of being a filtered version of $\frac{\beta}{\alpha} k^{i}\left(y-\hat{x}_{1}\right)$, thus recovering asymptotically the structure of the standard highgain observer (3) for low frequencies (in particular when $\alpha=\beta$ ). In the following we will also use the forthcoming compact notation, arising from the fact that $A$ is in the prime form (1),

$$
\begin{aligned}
\dot{\hat{x}} & =A \hat{x}+B \varphi_{s}(\hat{x})+\operatorname{diag}(L) \eta \\
\dot{\eta} & =-k\left(\alpha I-k \beta A^{T}\right) \eta+\beta k^{2} C^{T}(y-C \hat{x}),
\end{aligned}
$$

where the Luenberger gain $L$ is defined as in (3). While in the standard high-gain observer (3), the coefficients $\ell_{1}, \ldots, \ell_{n}$ need to satisfy the condition $(A-L C)$ Hurwitz, a more stringent condition is required for (8), as specified in the forthcoming section.

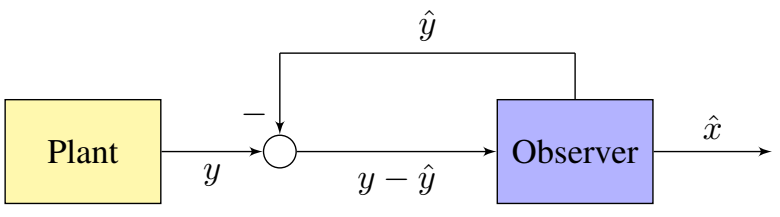

Fig. 1: Standard observer scheme. 


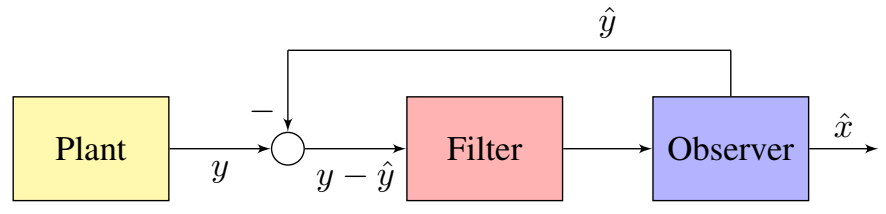

Fig. 2: Output injection redesign via dynamical filtering.

\section{B. A preliminary Lemma}

Let $\mathcal{S}_{\alpha, \beta} \subset \mathbb{C}_{<0}$ be the following set

$$
\mathcal{S}_{\alpha, \beta}:=\left\{(x+i y) \in \mathbb{C}: \alpha^{2} x+\beta y^{2}<0\right\},
$$

whose peculiar parabolic boundary is represented in Figures 3 and 5. We have then the following lemma instrumental to the main result of this section.

Lemma 1 Let two positive reals $\alpha, \beta$ be fixed and consider the following matrix

$$
M=\left(\begin{array}{c|c}
A & \operatorname{diag}(L) \\
\hline-\beta C^{T} C & -\alpha I+\beta A^{T}
\end{array}\right) .
$$

The following holds:

(i) The characteristic polynomial of $M$ satisfies

$$
p_{M}(\lambda)=\beta^{n} p_{(A-L C)}\left(\frac{\lambda(\lambda+\alpha)}{\beta}\right) .
$$

(ii) If $L$ is chosen such that $\sigma(A-L C) \subset \mathcal{S}_{\alpha, \beta}$, with $\mathcal{S}_{\alpha, \beta}$ defined in (9), then both $(A-L C)$ and $M$ are Hurwitz.

The proof of Lemma 1 is not detailed here due to space limits. The sketch of the proof is as follows. Relation (11) expresses the characteristic polynomial of $M$, of degree $2 n$, in terms of the characteristic polynomial of $(A-L C)$, of degree $n$, and parameters $\alpha$ and $\beta$. This relation can be obtained by iterative and suitable row and column expansions of the determinant $\operatorname{det}(M-\lambda I)$ and using the structure of $M$. The proof of item (ii) of Lemma 1 exploits equation (11), that is the link between the roots of $p_{M}(\cdot)$ and $p_{(A-L C)}(\cdot)$. Roughly speaking, we have $\lambda \in \sigma(M)$ if and only if $\frac{\lambda(\lambda+\alpha)}{\beta}=\mu \in \sigma(A-L C)$. The set $\mathcal{S}_{\alpha, \beta}$ defined by (9) is built as the intersection of two sets: the set of $\mu \in \sigma(A-L C)$ such that $\Re(\mu)<0$ and in addition the set of $\mu \in \sigma(A-L C)$ such that both roots $\lambda$ of the equation $\lambda(\lambda+\alpha)=\beta \mu$ verify $\Re(\lambda)<0$. We obtain the conclusion that if $\sigma(A-L C) \subset$ $\mathcal{S}_{\alpha, \beta}$, then both $(A-L C)$ and $M$ are Hurwitz.

Lemma 1 requires further comments. The design of $L$ such that both matrices $(A-L C)$ and $M$ be Hurwitz will start by selecting the spectrum $\sigma(A-L C) \subset \mathcal{S}_{\alpha, \beta}$ and in a second step by computing $L$ related to this desired spectrum by exploiting the companion structure of matrix $(A-L C)$. This procedure is detailed in Remark 1 at the end of this section.

We provide now two numerical examples illustrating the main features of Lemma 1.

Numerical example 1. Consider the case of a system of dimension $n=8$ and fix $\alpha=0.1$ and $\beta=0.1$. We select in this first example $L$ such that the eigenvalues of $(A-L C)$ lay inside the set $\mathcal{S}_{\alpha, \beta}$ defined in (9). In particular we choose $\sigma(A-L C)=\{-2.5 \pm 0.3 i,-2,-1.3,-1 \pm 0.2 i,-0.1,-0.4\}$.

Figure 3 shows the placement of the eigenvalues $(A-L C)$ and the parabola

$$
\alpha^{2} \Re(\mu)+\beta \Im^{2}(\mu)<0,
$$

corresponding to the boundary of set $\mathcal{S}_{\alpha, \beta}$. The eigenvalues of the closed-loop matrix $M$ in (10) are shown in Figure 4. It can be noticed that all the 16 eigenvalues have strictly negative real part and that they are symmetric with respect to the vertical axis $\Re(\lambda)=-\alpha / 2=-0.05$, highlighted in red in Figure 4. This symmetric placement of the eigenvalues $\lambda$ of $M$ is a direct consequence of property (i) of Lemma 1.

Numerical example 2. We select, as in the previous Example $1, n=8, \alpha=0.1$ and $\beta=0.1$. We select now $L$ such that two eigenvalues of $(A-L C)$ lay outside the set $\mathcal{S}_{\alpha, \beta}$ defined in (9). In particular we choose

$\sigma(A-L C)=\{-2.5 \pm 0.3 i,-2,-1.3,-1 \pm 0.6 i,-0.1,-0.4\}$.

Figure 5 shows the placement of the eigenvalues $(A-L C)$ and the parabola (12). It can be noticed that the eigenvalues in $-1 \pm 0.6$ are not inside the parabola defined by $\mathcal{S}_{\alpha, \beta}$. These two defective eigenvalues are highlighted in magenta squares. The eigenvalues of the closed-loop matrix $M$ in (10) are shown in Figure 6. It can be noticed that now 2 eigenvalues have positive real part, namely they are unstable. These eigenvalues are highlighted in magenta in Figure 6. Nevertheless, it can be remarked that the symmetry with respect to the vertical axis $\Re(\lambda)=-\alpha / 2=-0.05$, highlighted in red in Figure 6, is still respected.

\section{Asymptotic properties of the estimation error}

By direct application of Lemma 1 of the previous section, we obtain the following result.

Theorem 1 Consider system (2) and observer (7). Let $\alpha, \beta>0$ be fixed and let $L$ be chosen such that $\sigma(A-L C) \subset$ $\mathcal{S}_{\alpha, \beta}$. Then, there exists a real number $k^{*} \geq 1$, such that, for any $k>k^{*}$, the following bound holds

$$
|\hat{x}(t)-x(t)| \leq k^{n-1} \exp \left(-b_{1} k t\right) b_{2}+b_{3} k^{n-1} \sup _{t \geq 0}|\nu(t)|
$$

for all $t \geq 0$, for all initial conditions $(x(0), \hat{x}(0), \eta(0)) \in$ $X \times \widehat{X} \times E$ and for some constants $b_{i}>0, i=1,2,3$ independent of $k$.

Proof. By considering the change of coordinates $e:=$ $k D_{k}^{-1}(\hat{x}-x), \tilde{\eta}:=D_{k}^{-1} \eta$, and using $D_{k}^{-1} A=k A D_{k}^{-1}$ and $k D_{k}^{-1} A^{T}=A^{T} D_{k}^{-1}$, system (7) reads as

$$
\begin{aligned}
& \dot{e}=k A e+B \Delta(e, x)+k \operatorname{diag}(L) \tilde{\eta} \\
& \dot{\tilde{\eta}}=-k\left(\alpha I-\beta A^{T}\right) \tilde{\eta}-k \beta C^{T} C e+\beta k C^{T} \nu
\end{aligned}
$$

where $\Delta(e, x)$ is computed as

$$
\Delta(e, x):=k^{-(n-1)}\left[\varphi_{s}\left(D_{k} k^{-1} e+x\right)-\varphi_{s}(x)\right] .
$$


By setting $\varepsilon:=\operatorname{col}(e, \tilde{\eta})$, system (14) can be compactly written

$$
\dot{\varepsilon}=k M \varepsilon+\bar{B} \Delta(e, x)+k \beta \bar{C} \nu,
$$

where $M$ is given in (10), $\bar{B}:=\operatorname{col}(B, 0), \bar{C}=\operatorname{col}\left(0, C^{T}\right)$. Since $\varphi_{s}$ is globally bounded and $\varphi, \varphi_{s}$ are locally Lipschitz, we have that $|\Delta(e, x)| \leq \delta|e| \leq \delta|\varepsilon|$ for some $\delta>0$ for all $x \in X$ and all $e \in \mathbb{R}^{n}$. As a consequence, since the matrix $M$ is Hurwitz by construction (see in particular Lemma 1), by using the Lyapunvo function $V=\varepsilon^{T} P \varepsilon$, with $P=P^{T}>0$ solution of $P M+M^{T} P=-I$, and by applying standard Lyapunov arguments (see for instance [3] or [12]), which are here omitted for the sake of compactness, we can conclude that the following inequality

$$
|\varepsilon(t)| \leq \exp \left(-b_{1} k t\right) b_{2}+b_{3} \sup _{t \geq 0}|\nu(t)|,
$$

holds for all $t \geq 0$, for all $k>k^{*}$, where $k^{*} \geq 1$ is large enough, and for some $b_{i}>0, i=1,2,3$, independent of the parameter $k$. Finally, by using the previous bound and by recalling the inequality $|\hat{x}-x| \leq k^{n-1}|e| \leq k^{n-1}|\varepsilon|$ we obtain the bound (13), which concludes the proof.

Remark 1 A nice feature to be highlighted is the design of observer (7) and in particular how to choose the coefficients $\alpha, \beta, \ell_{1}, \ldots, \ell_{n}$ characterizing the gains of the observer. In particular, in view of Lemma 1, one can simply follow these steps:

- choose any $\alpha, \beta>0$;

- choose $n$ stable poles $\lambda_{i}$ (that can be real or complex conjugate) lying inside $\mathcal{S}_{\alpha \beta}$ of (9);

- compute the resulting characteristic polynomial $\prod_{i=1}^{n}\left(\lambda-\lambda_{i}\right)=\lambda^{n}+a_{1} \lambda^{n-1}+\ldots+a_{n-1} \lambda+a_{n}$; note that, in light of the Routh-Hurwitz necessary condition for the stability of a polynomial, we have $a_{i}>0$ for all $i=1, \ldots, n$;

- assign $\ell_{i}=a_{i}$ for all $i=1, \ldots, n$.

As it can be noticed, the procedure is substantially the one used to select the gain of a standard high-gain observer, but with the additional constraint that the poles of $(A-L C)$ lay inside $\mathcal{S}_{\alpha, \beta} \subset \mathbb{C}_{<0}$.

The proposed observer (7) ensures asymptotic convergence to zero of the estimation error $|\hat{x}-x|$. Even if the bound (13) has the same form as (4), the presence of the additional dynamics $\eta$ may improve the performances of the overall observer in the presence of measurement noise with respect to the standard high-gain observer (3). A detailed characterization of these performances is out of the scope of this preliminary work. However, we may note that the relative degree between the measured output $y$ and the estimation $\hat{x}$ is in general at least 2, so better performances in presence of high-frequency measurement noise are in general expected. In particular, by following the computation in [6], it is expected that when the measurement noise $\nu(t)$ is modelled as in (5), the asymptotic behaviour of the estimation error for (7) can be characterized by

$$
\lim _{t \rightarrow \infty}|\hat{x}(t)-x(t)| \leq \epsilon^{2} b_{4} k^{n} \sup _{t \geq 0}|\nu(t)|
$$

for some $b_{4}>0$ independent of $k$. We remark that in view of the special structure of the dynamical filter in (7), the bound (17) could be refined by inspecting all its components $\hat{x}_{i}-x_{i}$, $i=1, \ldots, n$, as done for instance in [6], [15]. However, because of space reasons, in this work we limit ourselves to show the effectiveness of the scheme in the numerical example in Section V.

Finally, we remark that the proposed structure (7) has the desirable feature of having the high-gain parameter $k$ which is powered up to 2 regardless of the dimension of the system state $n$. On the contrary, standard high-gain observers embed coefficients which are proportional to $k^{n}$. This feature may be of particular interest in case of a large state-dimension $n$ for numerical implementation purposes, as already highlighted in [12]-[16].

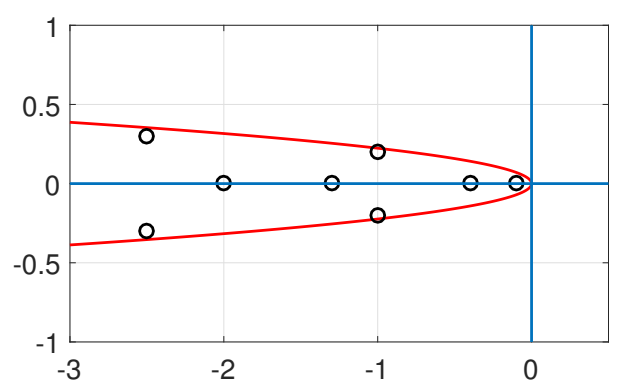

Fig. 3: Numerical example 1. Choice of the stable eigenvalues of $(A-L C)$.

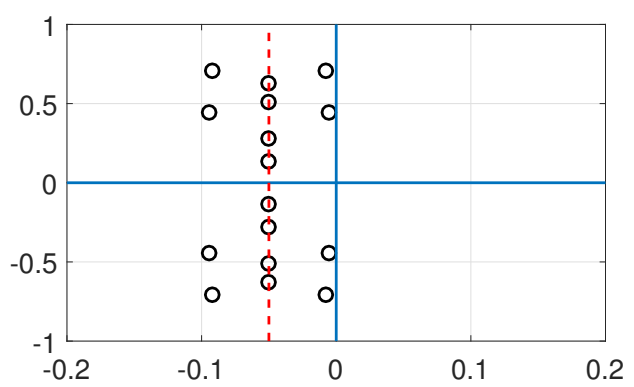

Fig. 4: Numerical example 1. Eigenvalues of the closed-loop matrix $M$ defined in (10).

\section{A General Formulation}

By following the general scheme of Figure 2, it can be noticed that the observer structure (8) presented in Section III can be further generalized as follows

$$
\begin{aligned}
\dot{\hat{x}} & =A \hat{x}+B \varphi_{s}(\hat{x})+D_{k} N H \eta, \\
\dot{\eta} & =k F \eta+k G(y-C \hat{x}),
\end{aligned}
$$

where $\hat{x} \in \mathbb{R}^{n}$ is the estimate of the state $x$ of (2), $\eta \in \mathbb{R}^{m}$ is the state of the filter, with $m \in \mathbb{Z}_{>0}$. We denote with $\widehat{X} \times E \subset \mathbb{R}^{n} \times \mathbb{R}^{m}$ the compact set of initial conditions of (18). Matrices $N, F, G, H$ are now degrees of freedom to be properly chosen. It is readily seen that the structure 


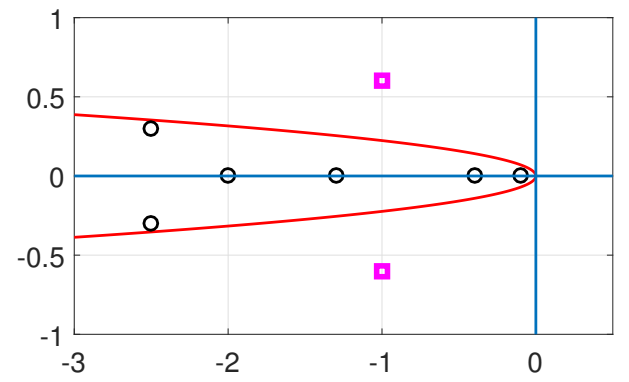

Fig. 5: Numerical example 2. Choice of the stable eigenvalues of $(A-L C)$.

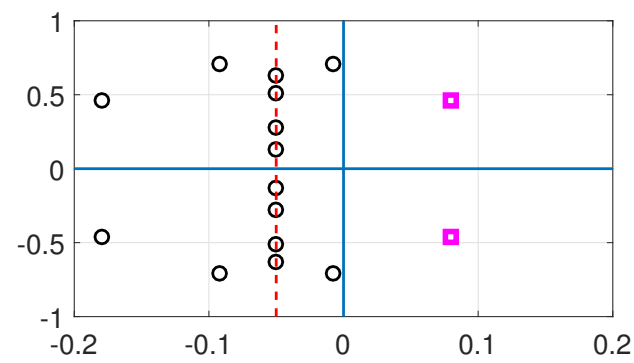

Fig. 6: Numerical example 2. Eigenvalues of the closed-loop matrix $M,(10)$. Two eigenvalues are unstable.

(8) is recovered from (18) by selecting $m=n, N=D_{k}^{-1}$, $H=\operatorname{diag}(L), F=\left(\alpha I-k \beta A^{T}\right)$ and $G=\beta k C^{T}$. By analysing the dynamics of system (3) and the observer (18), we can establish the following result.

Theorem 2 Consider system (2) and observer (18). Let $N, F, G, H$ be chosen such that the following matrix

$$
Q:=\left(\begin{array}{cc}
A & N H \\
-G C & F
\end{array}\right)
$$

is Hurwitz. Then, there exists a $k^{*} \geq 1$, such that, for any $k>k^{*}$, the following bound holds

$$
|\hat{x}(t)-x(t)| \leq k^{n-1} \exp \left(-b_{1} k t\right) b_{2}+b_{3} k^{n-1} \sup _{t \geq 0}|\nu(t)|,
$$

for all $t \geq 0$, for all initial conditions $(x(0), \hat{x}(0), \eta(0)) \in$ $X \times \widehat{X} \times E$ and for some constants $b_{i}>0, i=1,2,3$ independent of $k$.

Proof. By applying the change of coordinates $e:=$ $k D_{k}^{-1}(\hat{x}-x)$ and by denoting $\varepsilon:=\operatorname{col}(e, \eta)$, system (18) reads

$$
\dot{\varepsilon}=k Q \varepsilon+\bar{B} \Delta(e, x)+k \bar{G} \nu
$$

where $\bar{B}:=\operatorname{col}(B, 0), \bar{G}=\operatorname{col}(0, G)$ and $\Delta$ is computed as in (15). The end of the proof follows by following the same steps used in the proof of Theorem 1, and by recalling that the matrix $Q$ is Hurwitz by assumption.

Theorem 2 states that observer (18) guarantees asymptotic estimation of system (2) in nominal condition and moreover that the estimation error is input-to-state stable with respect to the measurement noise. This result may be seen as a generalization of Theorem 1. Furthermore, in view of the mild condition $Q$ Hurwitz, we believe that all the degrees of freedom, namely the dimension $m$ of the filter and the matrices $F, G, N, H$, could be selected in order to improve the behaviour of the observer in presence of measurement noise with some optimality criteria (deterministic or stochastic).

Remark 2 In this work we have not investigated the use of the new observer structure (18) in output feedback stabilization. However, in light of the properties highlighted in (20), it is expected that observers of the form (18) can be used in place of standard high-gain observers (3) without loss of generality since the same main features are retained (arbitrarily fast exponential convergence of the estimation error and input-to-state stability properties with respect to the measurement noise). Finally, we remark that also some robustness properties with respect to model uncertainties of the function $\varphi$ of the plant (2) are also expected, as in the case of standard high-gain observers.

\section{Simulation}

We consider the following nonlinear system

$$
\dot{x}=A_{4} x+B_{4} \varphi(x), \quad y=C_{4} x+\nu
$$

where $x \in \mathbb{R}^{4}$ is the state, $\left(A_{4}, B_{4}, C_{4}\right)$ is a triplet in prime form of dimension 4 , and the function $\varphi(x)$ is chosen as

$$
\varphi(x)=-x_{4}-5.2 x_{3}-2.7 x_{2}+4.5\left(x_{1}^{2}-1\right) .
$$

This system, for initial conditions $x=\operatorname{col}(0.9,0,0,0)$, has a chaotic attractor and bounded trajectories for all $t \geq 0$, see [19]. Furthermore, it can be numerically verified that solutions converge to a set where $\left|x_{i}(t)\right| \leq 6$ and $|\varphi(x(t))|<$ 25 for all $t \geq 0$. The measurement noise is modelled as $\nu(t)=\bar{\nu} \sin (\omega t)$ with $\bar{\nu}=0.1$ and $\omega \geq 50$.

We implement the filtered high-gain observer (7) by selecting $\varphi_{s}(x)=\operatorname{sat}_{25}(\varphi(x))$ where $\operatorname{sat}_{r}(s)=$ $\max (-r, \min (r, s)), \alpha=3, \beta=1$ and the poles of $(A-L C)$ are placed in $(-1 \pm i,-2 \pm i)$, which corresponds to selecting $L=\operatorname{col}(6,15,18,10)$. It can be verified numerically that the poles of $M$ are complex conjugates with real part placed between -2.7 and -0.3 . Asymptotic estimation is achieved in nominal conditions, namely when there is no measurement noise, for $k \geq 5$.

As a comparison, we run the simulation in the same conditions while considering a standard high-gain observer of the form (3). In this case, $L$ is taken as before, but we choose $k=4$ which is large enough to guarantee convergence of the observer in nominal conditions, namely when $\nu=0$. Figure 7 shows convergence of the estimation errors $|x(t)-\hat{x}(t)|$ of the two observers. For both observers the estimation error convergences asymptotically to zero with a convergence rate which is of the same order. It can be noticed that the presence of the filters in observer (7) attenuates the peaking phenomena during the transient.

Table I contains the data concerning the normalised asymptotic estimation errors

$$
\left\|\tilde{x}_{i}\right\|_{a}:=\limsup _{t \rightarrow \infty} \frac{\left|\hat{x}_{i}(t)-x_{i}(t)\right|}{\bar{\nu}}
$$


for various values of $\omega$ when $k=10$. Improvement of the performances is achieved when $\omega$ is taken higher. On the other hand it can be seen that the effect of the measurement on the last components of the estimation error is not amplified as in the case of standard high-gain observer. Finally, Table II shows the effect of the measurement noise on the asymptotic estimation errors (values are normalised with respect to $\nu$ as in Table I). It can be noticed that the measurement noise is amplified on the lower components and in general the estimation error provided by the filtered high-gain observer (7) has lower bounds (see Table I) even though the high-gain parameter is larger. An exact comparison between the two observers is however very hard to address since the dynamics are different because of the different dimension and eigenvalues of the closed-loop rescaled matrices $(A-L C)$ and $M$.

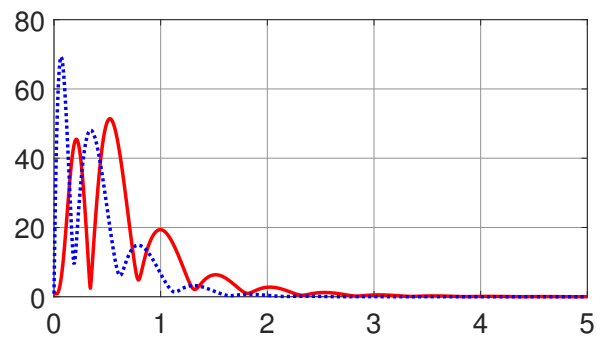

Fig. 7: Convergence of the estimation error $|x(t)-\hat{x}(t)|$. Dotted blue line: standard high-gain observer (3). Red line: high-gain observer with filtering redesign (7).

TABLE I: Normalized asymptotic estimation error (23) of the filtered high-gain observer (7).

\begin{tabular}{|l|l|l|l|}
\cline { 2 - 4 } \multicolumn{1}{c|}{} & $\omega=50$ & $\omega=100$ & $\omega=300$ \\
\hline$\left\|\tilde{x}_{1}\right\|_{a}$ & 2 & 0.6 & 0.07 \\
\hline$\left\|\tilde{x}_{2}\right\|_{a}$ & 10 & 1.4 & 0.07 \\
\hline$\left\|\tilde{x}_{3}\right\|_{a}$ & 22 & 1.6 & 0.02 \\
\hline$\left\|\tilde{x}_{4}\right\|_{a}$ & 18 & 0.8 & 0.01 \\
\hline
\end{tabular}

TABLE II: Normalized asymptotic estimation error (23) of the standard high-gain observer (3).

\begin{tabular}{|l|l|l|l|}
\cline { 2 - 4 } \multicolumn{1}{c|}{} & $\omega=50$ & $\omega=100$ & $\omega=300$ \\
\hline$\left\|\tilde{x}_{1}\right\|_{a}$ & 5 & 2.5 & 0.9 \\
\hline$\left\|\tilde{x}_{2}\right\|_{a}$ & 50 & 25 & 8 \\
\hline$\left\|\tilde{x}_{3}\right\|_{a}$ & 230 & 120 & 40 \\
\hline$\left\|\tilde{x}_{4}\right\|_{a}$ & 500 & 260 & 90 \\
\hline
\end{tabular}

\section{Conclusion}

We proposed to modify the standard high-gain observer structure by adding a dynamical filter processing the output injection term. We proved that the obtained observer inherits the asymptotic behaviour of the high-gain observer, namely asymptotic estimation can be achieved in nominal conditions, and input-to-state properties with respect to measurement noise are retained. We expect that the effect of augmenting the relative degree between estimates and measurements, and the choice of the dynamical filter, may improve the sensitivity with respect to measurement noise. This fact is confirmed by simulations although a rigorous proof is not provided. A particular choice of the filter allows to implement coefficients that are proportional to powers of the high-gain parameter equal to one or two, thus resulting in numerical benefits when the system's dimension is large or fast convergence is sought for (see [12], [16]).

This work proposes a different route for the problem of state estimation of nonlinear systems in the presence of measurement noise, which mainly combines a Luenberger observer with a dynamical filter in a feedback form (and not in cascade) and sufficient conditions for the stability of the composed observer are given. A full characterization of the behaviour of the proposed technique in presence of measurement noise and optimality-based tuning for both the gain and the parameters of the dynamical filter remains an open question for future research.

\section{REFERENCES}

[1] J.P. Gauthier and I. Kupka, "Deterministic observation theory and applications", Cambridge University Press, 2004.

[2] G. Besançon, "Nonlinear observers and applications", Springer, 2007.

[3] H. Khalil and L. Praly, "High-gain observers in nonlinear feedback control”, Int. J. Robust. Nonlinear Control, 24 (6), pp. 993-1015, 2014.

[4] L. K. Vasiljevic and H. K. Khalil, "Differentiation with high-gain observers the presence of measurement noise", IEEE 45th Conference on Decision and Control, pp. 4717-4722, 2006.

[5] A. A. Ball and H. K. Khalil, "High-gain observer tracking performance in the presence of measurement noise", American Control Conference, pp. 4626-4627, 2009.

[6] D. Astolfi, L. Marconi, L. Praly, A. Teel, "Sensitivity to High-Frequency Measurement Noise of Nonlinear High-Gain Observers", 10th IFAC Symposium on Nonlinear Control Systems - NOLCOS 2016, Monterey, California, USA, August 2016.

[7] J. H. Ahrens and H. K. Khalil, "High-gain observer in the presence of measurement noise: a switched-gain approach", Automatica vol. 45, pp 936-943, 2009.

[8] R. G. Sanfelice and L. Praly, "On the performance of high-gain observers with gain adaptation under measurement noise", Automatica vol. 47, pp. 2165-2176, 2011.

[9] N. Boizot, E. Buswelle, J.P. Gauthier, "An adaptive high-gain observer for nonlinear systems", Automatica, vol. 46, pp. 1483-1488, 2010.

[10] A. M. Boker and H. K. Khalil, "Nonlinear observers comprising highgain observers and extended Kalman filters", Automatica, vol. 49, pp. 3583-3590, 2013.

[11] D. Astolfi, R. Postoyan, D. Nešić, "Uniting local and global observers for the state estimation of nonlinear continuous-time systems", 56th IEEE Conference on Decision and Control, 2017.

[12] D. Astolfi and L. Marconi, "A High-Gain Nonlinear Observer with Limited Gain Power", IEEE Transactions on Automatic Control, Vol. 60 (11), pp. 3059-3064, 2015.

[13] A. Teel, "Further variants of the Astolfi/Marconi high-gain observer", American Control Conference 2016, Boston, USA.

[14] L. Wang, D. Astolfi, H. Su, L. Marconi, "High-gain observers with limited gain power for systems with observability canonical form", Automatica, vol. 75, pp. 16-23, 2017.

[15] D. Astolfi, L. Marconi, L. Praly, A. Teel, "Peaking-free low-power high-gain observers", Automatica, under revision.

[16] H. Khalil "Cascade high-gain observers in output feedback control", Automatica, vol. 80, pp. 110-118, 2017.

[17] D. Astolfi, A. Alessandri, L. Zaccarian, "Stubborn ISS Redesign for Nonlinear High-Gain Observers", IFAC 2017 World Congress, Toulouse, France

[18] H. Khalil "Analysis of the Use of Low-Pass Filters with High-Gain Observers", IFAC-PapersOnLine, vol. 49 (18), pp. 488-492, 2016.

[19] K.E. Chlouverakis and J. C. Sprott, "Chaotic hyperjerk systems", Chaos, Solitons \& Fractals, vol. 28, pp. 739-746, 2006. 